\title{
On Darboux-Treibich-Verdier potentials
}

\author{
A. P. Veselov \\ Department of Mathematical Sciences, Loughborough University, Loughborough, \\ LE11 3TU, UK and Moscow State University, Moscow, 119899, Russia \\ E-mail address: A.P.Veselov@lboro.ac.uk
}

To V.B. Matveev on his 65-th birthday

\begin{abstract}
It is shown that the four-parameter family of elliptic functions

$$
u_{D}(z)=m_{0}\left(m_{0}+1\right) \wp(z)+\sum_{i=1}^{3} m_{i}\left(m_{i}+1\right) \wp\left(z-\omega_{i}\right)
$$

introduced by Darboux and rediscovered a hundred years later by Treibich and Verdier, is the most general meromorphic family containing infinitely many finite-gap potentials.

Mathematics Subject Classifications: 34M05, 81R12

Keywords: finite-gap potentials, trivial monodromy
\end{abstract}

\section{Introduction}

In 1882 in a short Comptes Rendus article Darboux [5] introduced and studied the following differential equation as a generalisation of the famous Lamè equation:

$$
\begin{gathered}
-\frac{d^{2} y}{d z^{2}}+\left(\frac{m_{0}\left(m_{0}+1\right)}{s n^{2} z}+\frac{m_{1}\left(m_{1}+1\right) d n^{2} z}{c n^{2} z}+\frac{m_{2}\left(m_{2}+1\right) k^{2} c n^{2} z}{d n^{2} z}\right. \\
\left.+m_{3}\left(m_{3}+1\right) k^{2} s n^{2} z\right) y=\lambda y
\end{gathered}
$$

where $m_{i}, i=0,1,2,3$ are 4 integer parameters and $s n, c n, d n$ are the standard Jacobi elliptic functions with parameter $k$ (see [24]). In a more convenient Weierstrass form the family of the corresponding potentials is

$$
u_{D}(z)=m_{0}\left(m_{0}+1\right) \wp(z)+\sum_{i=1}^{3} m_{i}\left(m_{i}+1\right) \wp\left(z-\omega_{i}\right),
$$

where $\omega_{1}, \omega_{2}, \omega_{3}$ are the half-periods of the corresponding elliptic curve [24]. 
A hundred years later this family was rediscovered by Treibich and Verdier [21, 22] in the context of the finite-gap theory. Almost at the same time Inozemtsev [14] considered it in relation with the generalisations of the elliptic Calogero-Moser system.

The family (2) was known as Treibich-Verdier potentials until about 10 years ago V.B. Matveev pointed out that it was already in the old Darboux note [5]. Following [18] we will call it Darboux-Treibich-Verdier (DTV) family.

The aim of this note is to show that this family is actually a very special one: this is the most general linear family of meromorphic functions, which contains infinitely many finite-gap potentials (see a precise formulation below).

I did not see this claim in the literature but I would not be surprised if in some form it was known to the experts. I should say that the proof simply follows from the modern finite-gap theory in combination with the classical theory of differential equations in the complex domain.

This note was written on the occasion of 65-th birthday of Vladimir Borisovich Matveev, who was the first to appreciate and to demonstrate the importance of Darboux ideas for the modern theory of integrable systems.

\section{Claim and a proof}

In 1974 S.P. Novikov [19] made a remarkable discovery: he showed that a real periodic Schrödinger operator

$$
L=-D^{2}+u(z), D=\frac{d}{d z}
$$

having commuting differential operator

$$
A=D^{2 n+1}+a_{1}(z) D^{2 n-1}+\ldots+a_{2 n}(z)
$$

of an odd order $2 n+1$, has at most $n$ gaps in its spectrum (see also Lax [17]). Dubrovin [8] showed later that the converse is also true: for any such operator $L$ there exists an odd-order differential operator $A$ such that

$$
[L, A]=0 .
$$

Slightly abusing terminology, we will call any Schrödinger operator $L$ (not necessary real and in general with singularities) with this property and the corresponding potential $u(z)$ as finite-gap.

Note that the commutativity equation (3) is equivalent to nonlinear ordinary differential equation on $u(z)$, which is the stationary higher KdV flow [19]. Historically 
this equation was first investigated (without any relation to spectral theory) by Burchnall and Chaundy [2], who proved that the operators $L$ and $A$ satisfy the algebraic relation

$$
A^{2}=P(L)
$$

for some polynomial $P(x)$ of degree $2 n+1$. When the corresponding curve is nonsingular the potential can be expressed explicitly in the hyperelliptic theta-functions as it was shown by Its and Matveev [15]. Further development was due to Krichever, who introduced an important general notion of Baker-Akhiezer function [16].

The following property of the finite-gap operators will play crucial role in our considerations.

Painlevè property. All the finite-gap potentials $u(z)$ are meromorphic in the whole complex plane. The same is true for all solutions $\psi(z)$ of the corresponding Schrödinger equation

$$
-\psi^{\prime \prime}+u(z) \psi=\lambda \psi
$$

for all $\lambda$. In generic situation this follows immediately from Its-Matveev formulas [15], the general case see in Segal and Wilson [20].

Let $f_{0}(z), \ldots, f_{N}(z), z \in \mathbf{C}$ be some meromorphic functions and $V$ be finite-dimensional linear subspace spanned by them:

$$
V=\left\{u(z)=\sum_{i=0}^{N} \alpha_{i} f_{i}(z), \alpha_{i} \in \mathbf{C} .\right\}
$$

We will call such a subspace finite-gap if $V$ contains an infinite subset $K \subset V$ consisting of finite-gap potentials, such that $K$ is dense in $V$ in Zariski topology. This means that if a polynomial is vanishing at the points of $K$ then it must vanish identically. We also say that $V$ is maximal if $V$ is not contained in a larger finite-gap family.

Let $\wp(z)$ be the classical Weierstrass elliptic function with periods $2 \omega_{1}, 2 \omega_{2}$ and $\omega_{3}=\omega_{1}+\omega_{2}($ see $[24])$.

Theorem 2.1 Modulo shift in $z$ there are only 3 maximal finite-gap subspaces, consisting of the following elliptic, trigonometric and rational potentials respectively:

$$
\begin{gathered}
u_{e l l}(z)=\sum_{i=1}^{3} \alpha_{i} \wp\left(z-\omega_{i}\right)+\alpha_{4} \wp(z)+\alpha_{0}, \\
u_{\text {trig }}(z)=\alpha_{1} \frac{a^{2}}{\sin ^{2} a z}+\alpha_{2} \frac{a^{2}}{\cos ^{2} a z}+\alpha_{0}, \\
u_{\text {rat }}(z)=\alpha_{1} \frac{1}{z^{2}}+\alpha_{0} .
\end{gathered}
$$


The fact that the potentials $u_{e l l}(z)$ are finite-gap for parameters from the set

$$
K=\left\{\alpha_{i}=m_{i}\left(m_{i}+1\right), m_{i} \in \mathbf{Z}, i=1, \ldots, 4, \alpha_{0} \in \mathbf{C}\right\}
$$

is due to Treibich-Verdier [21]. Note that the set $K$ is indeed dense in $V \approx \mathbf{C}^{5}$ in Zariski topology. The trigonometric potentials

$$
u_{\text {trig }}(z)=\frac{m_{1}\left(m_{1}+1\right) a^{2}}{\sin ^{2} a z}+\frac{m_{2}\left(m_{2}+1\right) a^{2}}{\cos ^{2} a z}
$$

with integer $m_{1}, m_{2}$ sometimes are called Pöschl-Teller potentials, although they were studied already by Darboux [6]. They are known to be the result of Darboux transformations applied to $u=0$ (see e.g. [10]) and thus are finite-gap. The same is true about rational case when $\alpha_{1}=m(m+1)$ with integer $m$. Note that trigonometric and rational families can be considered as a limits of the elliptic family when one or both of the periods go to infinity.

Thus we must show only that there are no more maximal finite-gap subspaces. To prove this we need the following crucial fact, showing the relation of this question with groups generated by reflections.

Let $V$ be a finite-gap subspace. Consider its singular set $\Sigma=\Sigma(V)$, which is the union of the singularity sets (poles) of the corresponding functions $f_{0}, \ldots, f_{N}$. Let $z_{0} \in \Sigma$ be any such pole and consider the symmetry $s$ with respect to $z_{0}: s(z)=2 z_{0}-z$. We claim that $s(\Sigma)=\Sigma$ for any $z_{0} \in \Sigma$.

Proposition 2.2 The singular set $\Sigma(V)$ of any finite-gap subspace $V$ is symmetric with respect to each of its points. Moreover, every potential in $V$ is invariant under such a symmetry.

To prove this we use the Painlevè property of the finite-gap operators and Frobenius analysis of the corresponding Schrödinger equation $-\psi^{\prime \prime}+u(z) \psi=\lambda \psi$ in the complex domain.

Without loss of generality we can assume that $f_{0}=1$ and $z_{0}=0$ is the pole of $f_{1}(z)$ (all other $f_{i}$ can be assumed regular at 0 ). It is known that the finite-gap potentials have only second-order poles with zero residues (see e.g. [20]), so the same must be true for the functions $f_{1}, \ldots, f_{N}$. The Laurent expansion at zero of the potentials from $V$ has the form

$$
u(z)=\frac{c_{-2}}{z^{2}}+c_{0}+c_{1} z+c_{2} z^{2}+\ldots
$$

with $c_{-2}=\alpha_{1}$ and other $c_{i}=c_{i}\left(\alpha_{0}, \ldots, \alpha_{N}\right)$ are certain linear functions of the parameters. Let us consider the corresponding Schrödinger equation

$$
-\varphi^{\prime \prime}+u(z) \varphi=\lambda \varphi
$$


and ask when it has all solutions meromorphic in the vicinity of $z=0$ for all $\lambda$. In that case we will say that (10) has trivial monodromy around $z=0$.

The following important lemma looks classical, but as far as I know first appeared in Duistermaat and Grünbaum [9].

Lemma 2.3 [9]. The equation (10) has trivial monodromy around $z=0$ if and only if the Laurent coefficients of the corresponding potential (9) satisfy the conditions:

$$
c_{-2}=m(m+1), m \in \mathbf{Z}_{+}
$$

and

$$
c_{2 k-1}=0, k=1, \ldots, m
$$

The proof follows the classical Frobenius line: the solutions must be meromorphic, so

$$
\varphi=z^{-\mu}\left(1+\sum_{i=1}^{\infty} \xi_{i} z^{i}\right)
$$

Substituting this into equation and equating the coefficents we see that $\mu$ must satisfy the characteristic equation $\mu(\mu+1)=c_{-2}$, which implies (11). This is not enough yet since $\varphi$ may have a logarithmic term. A simple analysis [9] shows that the logarithmic terms are absent for all $\lambda$ if and only if in addition to (11) we have (12).

Now we can prove the proposition. Since finite-gap potentials have Painlevè property they have trivial monodromy. Thus we have infinitely many potentials (9) with parameters $c_{-2}=m(m+1)$ for infinitely many integers $m$. This means that we have all odd coefficients $c_{2 k-1}(\alpha)=0$ for the corresponding $\alpha=\left(\alpha_{0}, \ldots, \alpha_{N}\right) \in K$. Now because $K$ is Zariski dense this implies that $c_{2 k-1}=0$ for all potentials from $V$. This means that all the potentials from $V$ are even functions and hence their singularity set is symmetric with respect to $z_{0}=0$, which completes the proof.

Now we need the following elementary geometric

Lemma 2.4 Suppose that a discrete set $\Sigma \subset \mathbf{C}$ is invariant under reflection $z \rightarrow 2 z_{0}-$ $z$ with respect to every point $z_{0} \in \Sigma$. Then there are only 3 possibilities:

$$
\begin{gathered}
\Sigma=\left\{z=z_{0}+k_{1} \omega_{1}+k_{2} \omega_{2}, k_{1}, k_{2} \in \mathbf{Z}, \omega_{1} / \omega_{2} \notin \mathbf{R}\right\}, \\
\Sigma=\left\{z=z_{0}+k \omega, k \in \mathbf{Z}, \omega \neq 0\right\}, \\
\Sigma=\left\{z=z_{0}\right\} .
\end{gathered}
$$

The proof is simple: if we have two different points $z_{0}, z_{1} \in \Sigma$ then the composition of the symmetries with respect to them gives a shift $z \rightarrow z+2 \omega, \omega=z_{1}-z_{0}$. This means that the set must contain the one-dimensional lattice $\left\{z=z_{0}+k \omega, k \in \mathbf{Z}\right\}$. If we have more points then it is easy to see that we have either larger one-dimensional or two-dimensional lattice. The only remaining case is a one-point set. 
To derive our main result consider first the case (13) when $\Sigma$ is a shifted twodimensional lattice. The group generated by reflections with respect to the corresponding points acts on $\Sigma$ and has 4 orbits corresponding to the different parities of $k_{1}$ and $k_{2}$. The corresponding meromorphic functions are elliptic and must have the Darboux-Treibich-Verdier form (6) shifted by $z_{0}$. In the case (14) the potentials are trigonometric functions and according to Airault, McKean and Moser [1] must be of the general form

$$
u(z)=\sum_{i=1}^{N} \frac{m_{i}\left(m_{i}+1\right) a^{2}}{\sin ^{2} a\left(z-z_{i}\right)}+\text { const }, a=\pi / \omega .
$$

In order to fit (14) up to a shift in $z$ they must be of the form (7). The remaining rational case is similar. This completes the proof of Theorem 2.1.

Remark. As we can see from the proof Zariski density can be replaced by the condition that $K$ is not contained in any hyperplane in $V$.

\section{Concluding remarks}

What we saw here is another demonstration of a deep link between finite-gap property and trivial monodromy in the complex domain. The last property was extensively studied in the end of XIX-th century starting from the work by Hermite and Picard's work in 1870s. Darboux work [5] also appeared in this context. The famous S. Kowalevskaya's work on integrable case in rigid body dynamics was further development of this idea. A link with Kowalevskaya's work had been emphasized already at a very early stage of the modern finite-gap theory by Dubrovin, Matveev and Novikov in [7], the relation of Picard's work with elliptic finite-gap potentials was studied in detail by Gesztesy and Weikard [11].

I should mention that there are Schrödinger operators with trivial monodromy, which are not finite-gap. A simple example is given by

$$
u(z)=\frac{m(m+1)}{z^{2}}+P(z)
$$

for any even polynomial $P(z)$. For more interesting examples, including Painlevè-IV transcendents, we refer to [12, 13, 23]. Note that as it follows from the proof Proposition 2.2 is still valid if we replace finite-gapness by trivial monodromy property.

An obvious question is about multi-dimensional analogue of our result. It is natural to expect that the answer would be related to the reflection groups and should be given by the corresponding generalised quantum Calogero-Moser systems, see the elliptic case in [14, 3]. I would like to note also that the multi-dimensional trivial monodromy condition plays a key role in the theory of Huygens' principle [4]. 


\section{References}

[1] Airault, H., McKean, H.P., Moser, J. Rational and elliptic solutions of the Kortewegde Vries equation and a related many-body problem. Comm. Pure Appl. Math. 30 (1977), 95148.

[2] Burchnall J.-L., Chaundy T.W. Commutative ordinary differential operators. Proc. London Math. Soc. 21 (1923), 420-440; Proc. Royal Soc. London (A) 118 (1928), 557-583.

[3] Chalykh O., Etingof P., Oblomkov A. Generalized Lamè operators. Comm. Math. Physics. 239 (2003), no 1-2, 115-153.

[4] Chalykh O.A., Feigin M.V., Veselov A.P. Multidimensional Baker-Akhiezer functions and Huygens' principle. Comm. Math. Physics, 206 (1999), 533-566.

[5] Darboux G. Sur une équation linéare. C. R. Acad. Sci. Paris, t. XCIV (1882), no. $25,1645-1648$.

[6] Darboux G. Lecons sur la théorie des Surfaces. Vol. 2. Paris: Gauthier-Villars, 1915. pp 210-215.

[7] Dubrovin B.A., Matveev V.B., Novikov S.P. Non-linear equations of the $K d V$ type, finite-gap linear operators and abelian varieties. Russian Math.Surveys, 31 (1976), 55-136.

[8] Dubrovin B.A. Periodic problems for the Korteweg-deVries equation in the class of finite-gap potentials. Functional Anal. Appl., 9 (1975), 215-223.

[9] Duistermaat, J.J., Grünbaum, F.A. Differential equations in the spectral parameter. Commun. Math. Phys. 103 (1986), 177240.

[10] Gaillard P., Matveev V.B. Wronskian and Casorati determinant representations for Darboux-Pöschl-Teller potentials and their difference analogue. J. Phys. A: Math. Theor. 42 (2009).

[11] Gesztesy F. and Weikard R. Picard potentials and Hill's equation on a torus. Acta Math., 176 (1996),73-107.

[12] Gibbons J., Veselov A.P. On the rational monodromy-free potentials with sextic growth. J. Math. Phys., Vol. 50 (2009), No.1, 013513.

[13] Hemery A.D., Veselov A.P. Whittaker-Hill equation and semifinite-gap Schrödinger operators. arXiv:0906.1697(2009). To appear in JMP.

[14] Inozemtsev V.I. Lax representation with spectral parameter on torus for integrable particle systems. Lett. Math. Phys., 17 (1989), 11-17. 
[15] Its A.R., Matveev V.B. Schrödinger operators with finite-gap spectrum and Nsoliton solutions of the Korteweg-de Vries equation. Theor. Math. Phys., 23 (1975), 343-355.

[16] Krichever I.M. Methods of algebraic geometry in the theory of non-linear equations. Russian Math. Surveys 82 (6) (1977) , 185-213.

[17] Lax P.D. Periodic solutions of the KdV equation. Commun. Pure Appl. Math., 28, No. 2 (1975), 141-188 .

[18] Matveev V.B., Smirnov A. On the link between the Sparre equation and Darboux-Treibich-Verdier equation. Lett. Math. Phys., 76 (2006), pp. 283-295.

[19] Novikov S.P. The periodic problem for the Korteweg-deVries equation. Functional Anal. Appl., 8 (1974), 236-246.

[20] Segal G. and Wilson G. Loop groups and equations of KdV type. Publ. Inst. Hautes Etudes Sci. Publ. Math., 61 (1985), 5-65.

[21] Treibich, A., Verdier, J.-L. Solitons elliptiques. Progr. Math. (The Grothendieck Festschrift, vol. 3). v.88. Birkhäuser-Boston, Boston, pp. 437480 (1990).

[22] Verdier J.-L. New elliptic solitons. In Algebraic Analysis (M. Kashiwara and T. Kawai, eds.), pp. 901-910. Academic Press, Boston, 1988.

[23] Veselov A.P. On Stieltjes relations, Painlevé-IV hierarchy and complex monodromy. J. Phys A.: Math and Gen., 34 (2001), 3511-3519.

[24] Whittaker E.T., Watson G.N. A Course of Modern Analysis. Cambridge Univ. Press, 1963. 